\title{
Erratum to "Piketty's Inequality between the Profit and Growth Rates and Its Implications for the Reproduction of Economic Elites" [Theoretical Economic Letters, 6, (2016) 1363-1392]
}

\author{
Alberto Benítez Sánchez \\ Economics Department, Universidad Autónoma Metropolitana, México City, México \\ Email: abaxayacat13@gmail.com
}

Received: September 15, 2017

Accepted: October 23, 2017

Published: October 26, 2017

Copyright $\odot 2017$ by author and Scientific Research Publishing Inc. This work is licensed under the Creative Commons Attribution International License (CC BY 4.0).

http://creativecommons.org/licenses/by/4.0/
In Benítez Sánchez, A., Piketty's Inequality between the Profit and Growth Rates and Its Implications for the Reproduction of Economic Elites. Theoretical Economic Letters 6, 1363-1392. http://dx.doi.org/10.4236/tel.2016.66125 each one of Examples 1, 2 and 7 contains a different error. This addendum points out the errors and presents the corrected versions of the examples.

The detail of the errors is as follows. Contrarily to what is assumed respectively in Examples 1, 2 and 7, Equation (31) produces the ratio $\frac{1+m_{t}}{1+g_{t}}$ (not $\frac{m_{t}}{g_{t}}$ ), Equation (44) produces the ratio $\frac{1+\mu_{t}}{1+g_{t}}$ (not $\frac{\mu_{t}}{g_{t}}$ ) and Equation (172) must be satisfied by the growth rate of $\beta_{t}$ (not by $m_{t}$ ). For these reasons, the part of each Example starting with the corresponding equation is not valid. It is important to add that the content of the paper other than the three examples is not affected by these errors, for which I apologize to the readers. The corrected versions of the examples come next.

On page 1369:

Example 1. According to Table S3.2 by Piketty [6] national capital measured by national income in France was equal to 3.51 in 1970 and, starting from this year increased continuously reaching 6.05 in 2010 . Thus, substituting by its corresponding values the variable $\beta_{t}$ in Equation (36) by Benítez [2] yields $A P K_{1970}=\frac{4.51}{3.51}$ and $A P K_{2010}=\frac{7.05}{6.05}$. Furthermore, it follows from Equation 
(49) by Benítez [2] that, if the annual rate of change $\left(h_{t}\right)$ of the $A P K$ had been steady between the two dates considered, then $A P K_{1970}\left(1+h_{t}\right)^{40}=A P K_{2010}$. Substituting in this equation $A P K_{1970}$ and $A P K_{2010}$ by their corresponding values gives $\frac{4.51}{3.51}\left(1+h_{t}\right)^{40}=\frac{7.05}{6.05}$, which implies that

$h_{t}=\left(\frac{3.51 \times 7.05}{4.51 \times 6.05}\right)^{\frac{1}{40}}-1=-0.002439$. On the other hand, according to Table 5.1 by Piketty [6], during the same period the national income growth rate in France was $2.2 \%$. Thus, the national income of 2010 was $(1+0.022)^{40}$ times that of 1970. This result plus the fact that $\beta_{2010}=6.05$, taken together, imply that the national capital of 2010 was $6.05(1+0.022)^{40}=14.44745$ times the national income of 1970. In turn, this result plus the fact that $\beta_{1970}=3.51$, taken together, imply that, if the capital growth rate had been steady during the period considered, it would satisfy the equation $3.51\left(1+m_{t}\right)^{40}=14.44745$ from which it follows that $m_{t}=\left(\frac{14.44745}{3.51}\right)^{\frac{1}{40}}-1=0.036005$. Now, substituting $h_{t}$ and $m_{t}$ by their corresponding values in Equation (54) by Benítez [2] gives:

$$
g_{t}=0.036005-0.002439+0.036005 \times(-0.002439)
$$

Therefore, $g_{t}=0.033477$. Due to the increase in the capital/income ratio, the capital growth rate exceeded the economic growth rate in $3.6005-3.3477=0.2528$ percentage points which represents the fraction $\frac{0.002528}{0.033477}=0.075514$ of the second rate. Hence, during the period considered, the average value of $m_{t}$ was approximately $7.55 \%$ greater than that of $g_{t}$.

\section{On page 1370:}

Example 2. According to Example 1, during the period 1970-2010, the average value of the economic growth rate was $g_{t}=3.3477 \%$ while, according to Table 5.1 by Piketty [6] during the same period the national income growth rate in France was $\mu_{t}=2.2 \%$. Therefore, due to the increase in the capital/income ratio the economic growth rate exceeded the national income growth rate in $3.3477-2.2=1.1477$ percentage points which represents the fraction $\frac{0.011477}{0.022}=0.521681$ of the second rate. Hence, during the period considered, the average value of $g_{t}$ was approximately $52.16 \%$ greater than that of $\mu_{t}$.

\section{On page 1388:}

Example 7. Let $r_{f}$ be the average profit rate (after taxes) obtained by the capital owned by consumer $f$ during the period separating date $t$ from date $t+\frac{n_{1}}{2}$. If $r_{f}$ satisfies inequality (167), doing the corresponding substitutions in this inequality with the data from Examples 1 (from this Erratum) and 6 yields:

$$
r_{f}>2^{\frac{1}{35}}(1+0.036005)-1
$$


$\Rightarrow$

$$
r_{f}>0.056726
$$

Thus, if the profit rate (after taxes) of an individual capital is greater than $5.6726 \%$, the owner of that capital may be a quasi-feudal renter if the capital is big enough, which depends on the actual value of $r_{f}$. For instance, if $r_{f}=7 \%$, substituting the corresponding data in the right-hand side of inequality (168) gives:

$$
s_{t f} \geq \frac{2^{\frac{1}{35}}(1+0.036005)-1}{0.07}
$$

$\Rightarrow$

$$
s_{t f} \geq 0.810382
$$

Therefore, to satisfy condition (162) it is enough owning a capital sufficiently large to live with $18.96 \%$ or less of what is obtained as profit. Now, substituting $s_{t f}$ in the right-hand side of Equation (155) by its minimum value according to inequality (175) gives:

$$
\begin{aligned}
I_{t f} & =\frac{y_{t_{0}}}{1-(0.810382)^{\frac{1}{2}}} \\
& =\frac{y_{t_{0}}}{0.099787} \\
& =10.021345 y_{t_{0}}
\end{aligned}
$$

This equation indicates a sufficient annual income. The corresponding capital is:

$$
\begin{aligned}
K_{t f} & =\frac{10.021345}{0.07} y_{t_{0}} \\
& =143.162078 y_{t_{0}}
\end{aligned}
$$

Accordingly, if an inherited capital obtains a profit rate of 7\% during 35 years, it is sufficient that the capital be equal to or greater than the quantity indicated in Equation (180) for its owner to be a quasi-feudal rentier.

In addition to the preceding examples, there are also the following errata.

1) On page 1375, just above Equation (83), where it says "... data from examples 2 and $3, \ldots$ " it should say "... data from examples 1 and $3, \ldots$ "

2) On page 1386, one line above Equation (150), where it says " $r=0.05385$ and, ..." it should say " $r=0.05385$ (after taxes) and, ..."

3) On page 1391 , line 5 , where it says "... may occur both if this ratio increases as if it ..." it should say "... may occur as well if this ratio increases as if it ..." 